\title{
Kinetic profile of silver and zinc oxide nanoparticles by intraperitoneal injection in mice , a comparative study
}

\author{
Sarab Mohammed M. Razooki ${ }^{1}$, Adel M. Rabee ${ }^{2}$ \\ ${ }^{1}$ Iraqi Accreditation Authority, Ministry of Planning \\ ${ }^{2}$ University of Baghdad, College of Science, Biology Department
}

\section{Article Info}

Received, 2019

\section{Keyword:}

kinetic

Ag NPs

ZnO NPs

Intraperitoneal

ADME

\begin{abstract}
To investigate the kinetic profile (Absorption, distribution, and excretion ) of intraperitoneal injected Ag NPs $(24.52 \mathrm{~nm})$ and ZnO NPs $(25.16 \mathrm{~nm})$ in Albino mice , a single dose of the two kinds of NPs $(100 \mathrm{mg} / \mathrm{kg})$ were used for comparison .Kinetic profile in blood clarified shorter time ( $\mathrm{T}$ max ) were spend by $\mathrm{ZnO}$ NPs to be absorbed compared with longer one recorded by $\mathrm{Ag}$ NPs as their smaller volume of distribution (Vd) to confirm their higher concentration (C max ) with $95 \mu \mathrm{g} / \mathrm{ml}$ compared with Ag NPs which stands on $57.5 \mu \mathrm{g} / \mathrm{ml}$, also Ag NPs needs longer time of elimination to their half values (t1/2 Elem.) in blood with lower clearance rate (CL) compared with ZnO NPs . This study discussed the distribution profile in different organs over time and pointed a considerable Ag and $\mathrm{Zn}$ concentrations specially in liver, spleen, intestine and kidney, also an important levels recorded in lung ,heart, testes and brain. $\mathrm{Ag}$ and $\mathrm{ZnO}$ NPs excretion manner noted a significant percentage of excretion via feces with $33 \%$ and $83 \%$ for Ag NPs and ZnO NPs respectively compared with small percentage recorded with urine .
\end{abstract}

\section{Corresponding Author:}

Sarab Mohammed M. Razooki

Iraqi Accreditation Authority

Ministry of Planning, Iraq

Al- Jadriya - Baghdad- Iraq

Email: sarabmohammad100@yahoo.com

\section{Introduction}

Nano industry has been described as the next industrial revolution as turning to be one of the fastest growing industries in the history of human beings [1] with expanding nanotechnology scope in continuous manner as using of nano materials in many implementations in our daily life [2]. Nanoparticles are defined as one or more dimension small objects in range of 1-100 $\mathrm{nm}$. Due to their small size and large- scale surface area compared with micro scale, different degrees of biological toxic effects were demonstrated by large numbers of studies [3]-[6]. Silver nanoparticles (Ag NPs) with mean annual production of 55 tons, individually represent the highest used in many consumer products with the widely expansion in NPs commerce which are used as antimicrobial agent in different consumer products with range from clothing, cosmetics, shoes, respirators surface coating, detergents, water filters and house purification systems, laptops, and phones [7] . Among metal containing nanoparticles, zinc oxide (ZnO NPs) was the third highest production over the world with 550 tons, widely used in cosmetic products as UV light scatter specially in sun screens, and dermal ointments, and toothpastes [8]. ZnO NPs mostly used in solar cells production and LCDs pigments, electronics, rubber, textiles and chemical fiber production [9],[10]. Due to the high surface-to-volume ratio, greater oxidant capacity and bio persistence, nanoparticles became the primary source for toxicity, which can penetrate easily through epithelium and reach to interstitial pulmonary area. There are real needs to protect our public and environmental health and safety, specially where the standards or guidelines that can directly rule 
nano materials effects are not exist in present time [11]. However, the tunable and varied physicochemical properties of Nanoparticles pose a new challenge for understanding their biological behavior, and bio distribution, so the in vivo and in vitro kinetics can be controlled to meet the requirements of efficient drug delivery and minimizing side-effects. In vivo porticokinetics refers to the absorption, distribution, metabolism, and excretion (ADME) of nanomaterials. the wide definition for Toxicikinetics described by [12] as the movement and fate, also referred to as the disposition, of toxicants. The term is most commonly used when describing the time course of absorption, distribution, and elimination (including biotransformation and excretion) of toxicants in an organism. blood concentrations analysis against time course after single dose administration of Ag NPs and ZnO NPs was efficient method for evaluate the amount of absorption and quantitative the availability, and helps in evaluation the amount of distribution and elimination steps [13]. Many studies described kinetic behavior of $\mathrm{Ag}$ and $\mathrm{ZnO}$ NPs followed oral or intravenous administration [14]-[17] and Only single study demonstrated the biodistribution of $\mathrm{ZnO}$ NPs over $27 \mathrm{~h}$ followed intraperitoneal injection. But no occurred any study demonstrate and analyze the kinetic parameters and behaves of NPs followed intraperetoneal injection of $\mathrm{Ag}$ or $\mathrm{ZnO}$ NPs in mice at world level, in addition to the comparison between them.

\section{Materials and Methods}

\subsection{Nanoparticles}

Un coated Ag NPs (20 nm as the specification sheet ) were purchased as grey to black nano powder from NANOSHEL company - USA, with purity of $99 \%, 10.5 \mathrm{~g} / \mathrm{cm} 3$ density , and spherical morphology . ZnO NPs (10-30 nm as the specification sheet ) were purchased as white to light yellow nano powder from Skyspring Nanomaterials incorporation -USA, with purity of $99.8 \%, 5.606 \mathrm{~g} / \mathrm{cm} 3$ density and spherical morphology. Ag and ZnO NPs were characterized using scanning probe microscope (SPM) from FILIPSGermany to determine size average for the two kinds of nanoparticles that used in this study. The surface morphology of the Ag NPs and ZnO NPs were displayed by atomic force microscope (AFM) under normal atmospheric conditions. The examined samples of nanoparticles were dispersed on glass slide and explored using the instrument.

\subsection{Animals housing}

Healthy adult male (8-10 week aged) Swiss albino mice with average weight $25 \pm 2$ gm were purchased from the national center for drug control and research - Ministry of Health. All mice were housed in polypropylene cages under controlled conditions of temperature $25 \pm 5^{\circ} \mathrm{C}$, humidity of 50-60\%, and $12 \pm 2$ hours light/dark cycles. Standard diet pellet and water ad libitum were used for feeding. The animals were kept for 7 days before starting the experiments for acclimatization to laboratory conditions. All animals were dealt in accordance to the guidelines of the Care and Use of Laboratory Animals- National Research Council and in accordance with the guidelines of the international guidelines for animal experimentation.

\subsection{Ag and ZnO NPs suspensions preparation}

Concentration $(100 \mathrm{mg} / \mathrm{kg})$ of Ag NPs and ZnO NPs suspensions were prepared with deionized distilled water . The two suspensions were homogenized by vortex for $20 \mathrm{sec}$, then exposed to probe ultrasound sonication from Soniprep 150 MES -UK (pulsed mode of $1 \mathrm{~min}$ operation and $30 \mathrm{sec}$. stopping ) in ice bath for $60 \mathrm{~min}$ [18], the prepared suspensions were immediately exposed to mice with single intraperitoneal injection.

\subsection{Experimental desigin}

Three groups of male albino mice with average weight $25 \pm 2$ gm used for kinetic study, each group contain 34 mice, first group intraperitoneally injected with single dose of $100 \mathrm{mg} / \mathrm{kg} \mathrm{Ag} \mathrm{NPs}$, the same dose of $\mathrm{ZnO}$ NPs for the second group, the third group was control. Samples of $1 \mathrm{ml}$ blood, and 0.25-0.5 gm of organs tissues including (liver, spleen, kidney, intestine, brain, testis, lung, heart) collected from two mice at specific time points after injection $(5,10,15,30,60 \mathrm{~min}, 2,4,6,24 \mathrm{~h}, 2,4,7,9,11,14,18 \mathrm{~d})$ and kept in $-20 \mathrm{C}^{\mathrm{o}}$ until prepared for digestion. Feces and urine samples collected from each group daily. 


\subsection{Ag and ZnO NPs quantitative analysis}

Sub samples 0.25-0.5 gm of tissue (liver, spleen, kidney, intestine, brain, testis, lung, heart), $1 \mathrm{ml}$ of whole blood, urine, and $1 \mathrm{gm}$ feces samples digest individually using microwave digestion system from Milestone Italy as described in [19],[20] using $9 \mathrm{ml}$ of $68-72 \%$ nitric acid $\mathrm{HNO} 3,1 \mathrm{ml}$ of $37 \%$ hydrochloric acid $\mathrm{HCl}$, and $2 \mathrm{ml}$ of $30 \%$ hydrogen peroxide $\mathrm{H} 2 \mathrm{O} 2$, the digested samples diluted to $25 \mathrm{ml}$ using distilled water. The concentration of silver and zinc detect in each sample using flame or flameless atomic absorption spectrophotometer (AAS) from Shimadzu- Japan.

\subsection{Kinetic analysis}

The kinetic analysis was performed using a non compartmental model . The bio kinetic parameters including maximum concentration ( $\mathrm{C}$ max), Time to achieve maximum concentration ( $\mathrm{T}$ max), Half-life of absorption (T1/2 Abs) , Half-life of Elimination (T1/2 Elem) calculated using the equation $\mathrm{T} 1 / 2=0.693 / \mathrm{k}$, were the elimination rate constant (K Elem) estimated using the formula $\mathrm{k}=-2.303 \times((\log$ conc.2- $\log$ conc. 1$) /($ time2time1)), Clearance rate (CL) $=\mathrm{K} \times \mathrm{Vd}$, and apparent Volume of distribution (Vd) was determined from the intercept of the curve. $\mathrm{V} \mathrm{d}=$ Dose/Intercept as described by [21].

\subsection{Statistical analysis}

The results are presented as the mean \pm standard error of means (SE). Analysis of variance (ANOVA) and least significant difference (LSD) were used to explain the differences between means over time points in feces and urine samples at $(\mathrm{p} \leq 0.05)$.

\section{Results and descussion}

\subsection{Characterization of Ag and ZnO NPs}

According to the granularity distribution chart , the average diameter for $\mathrm{Ag}$ and $\mathrm{ZnO}$ NPs samples were 24.52 $\mathrm{nm}, 25.16 \mathrm{~nm}$ respectively with spherical shape determined for the two types of NPs using AFM (Figure 1)

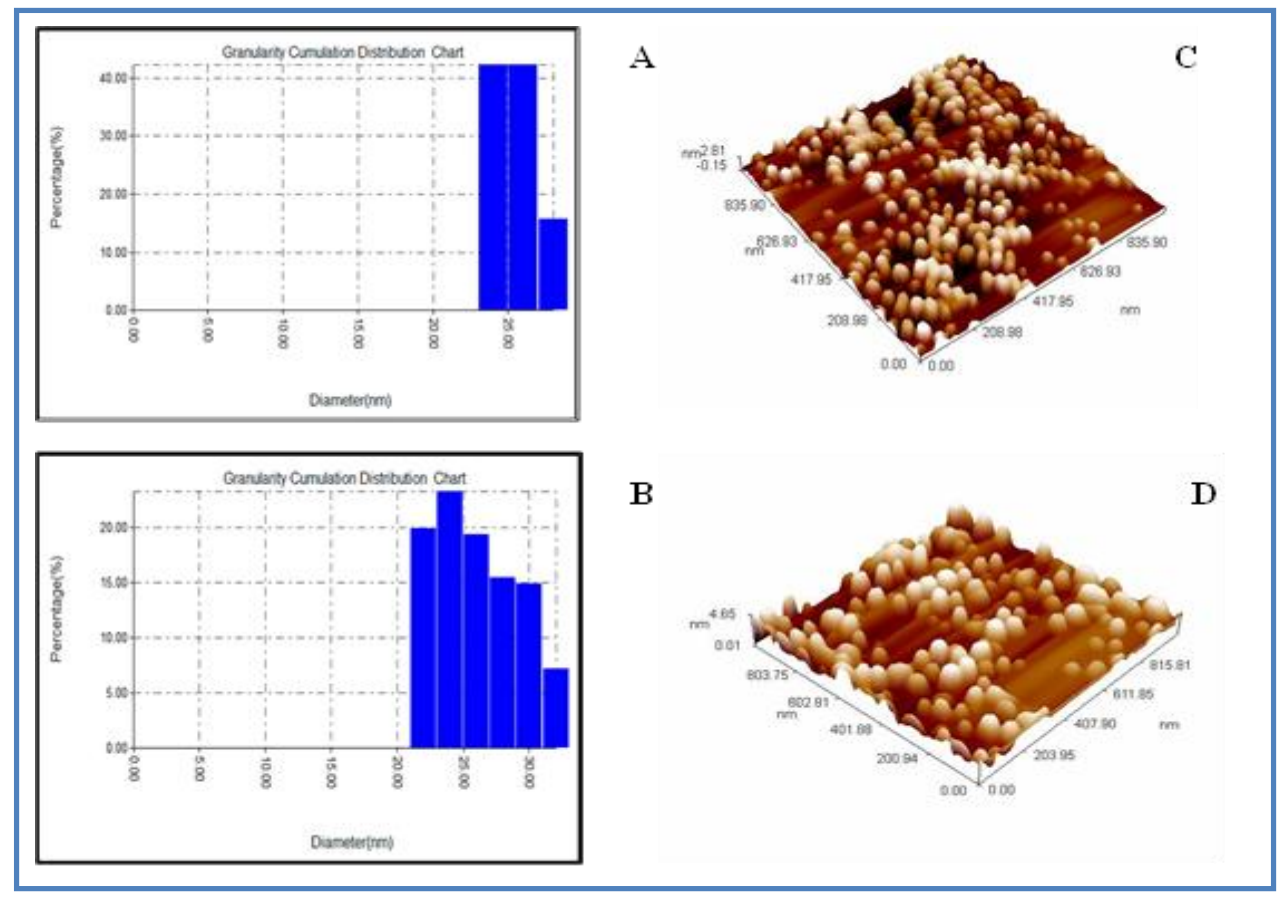

Figure 1. Characteristics of $\mathrm{Ag}$ and $\mathrm{ZnO}$ NPS , granularity distribution chart (left) for AgNPs (A) and $\mathrm{ZnO}$ NPs (B), spherical shape using AFM (right) for Ag NPs (C) and ZnO NPs (D). 


\subsection{Ag and ZnO NPs absorption}

Blood concentrations analysis against time course after single dose administration of Ag NPs and ZnO NPs was efficient method for evaluate the amount of absorption and quantitative the availability, and helps in evaluation the amount of distribution and elimination steps [13]. Highly significant difference $(\mathrm{p} \leq 0.05)$ in blood silver and $\mathrm{Zn}$ concentrations were illustrated over different time points (Fig 2 , table 1). The concentrations elevated within first few minutes followed injection with absorption half life (T 1/2 Abs) of $26.32 \pm 0.57 \mathrm{~min}$ and $14.30 \pm 0.00 \mathrm{~min}$ for $\mathrm{Ag}$ and $\mathrm{Zn}$ respectively, and recording maximum concentration $\mathrm{C}$ max. with $57.50 \pm 2.50 \mu \mathrm{g} / \mathrm{ml}, 95 \pm 0.00 \mu \mathrm{g} / \mathrm{ml}$ after $1 \mathrm{~h}$ and $30 \mathrm{~min}$ respectively which indicate the shorter needed time for $\mathrm{ZnO}$ absorption in versus with Ag NPs by the effect of the low molecular weight of zinc, and the effect of lower apparent volume of distribution $(\mathrm{Vd})$ compared with silver by which $\mathrm{ZnO}$ NPs could quickly reach distribution equilibrium between blood and tissues [22]. Lower value of maximum concentration detected in blood referred to silver compared with zinc which may caused by molecular weight variation [24], the probable aggregations of Ag NPs as the postponement in the peritoneal cavity leading to increase the surface area [23] and reduce the amount of silver that can pass through peritoneal membrane , in addition to and amount of generated ions [21], [25] and some considerable effects like lymphatic drainage , peritoneal permeability, and particle charge [26] , [27] .

The results of current study approach with those of previous one carried out by [29] showed the elevation of blood zinc level after $30 \mathrm{~min}$. of intraperitoneal injected with $2.5 \mathrm{~g} / \mathrm{kg} \mathrm{ZnO}$ NPs with $>100 \mathrm{~nm}$ in diameter, the peak point recorded after $6 \mathrm{~h}$, then kept with equilibrator scale at $72 \mathrm{~h}$ point . In the current study blood zinc concentrations need less time to reach their peak point as the effect of smaller particle size and lower concentration that play an important role in peritoneal particokinetic . Shorter half life of elimination (T1/2 Elem) recorded for zinc oxide NPs $2.38 \pm 0.08 \mathrm{~d}$ from blood compared with silver $8.39 \pm 0.23 \mathrm{~d}$ which considered long and suggested that Ag NPs can not removed from the body in short time (table 2), this slow elimination manner with low clearance rate $(\mathrm{Cl})$ of $0.43 \pm 0.03 \mathrm{mg} / \mathrm{d} . \mathrm{kg}$ compared with higher recorded one with ZnO NPs $0.87 \pm 0.03 \mathrm{mg} / \mathrm{d}$.kg explained by formation silver protein complexes specially with sulfhydryl containing proteins as the high affinity of silver for sulfur group [14],[28] , also the insoluble part of two types of NPs may involved. The calculated apparent volume of distribution (Vd) were $5.16 \pm 0.251 / \mathrm{kg}(129.00$ $\pm 6.15 \mathrm{ml} / 25 \mathrm{gm})$ and $2.99 \pm 0.02 \mathrm{l} / \mathrm{kg}(74.75 \pm 0.56 \mathrm{ml} / 25 \mathrm{gm})$ for $\mathrm{Ag}$ and $\mathrm{ZnO}$ NPs respectively, with taking

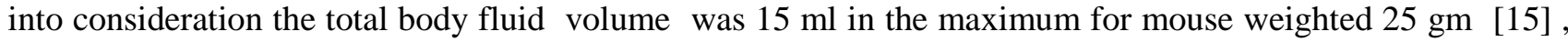
smaller volume of distribution of $\mathrm{ZnO}$ NPs illustrated the shorter needed time for distribution equilibrium [21] . It is worth to mention that the resulted values of kinetic parametersb of current study should not be compared with parameters in the other studies as the differentr mathematical models and animals weret used in the currents study.
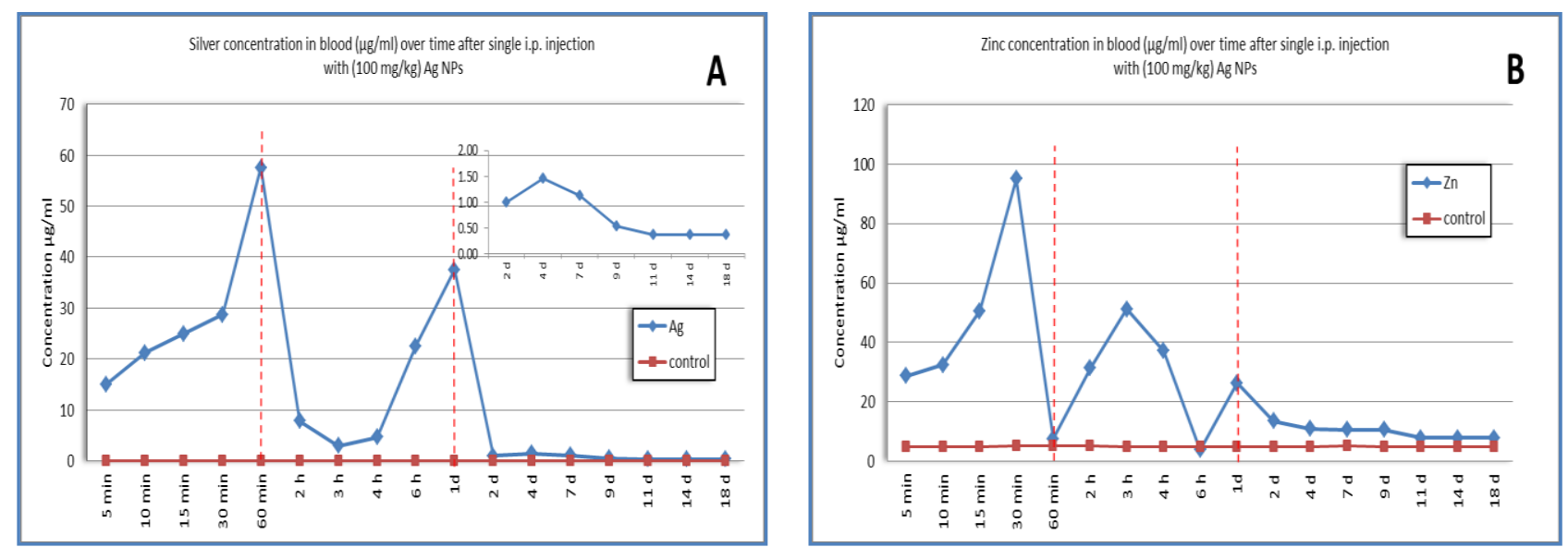

Figure 2. Silver (A) and Zinc (B) concentrations means in blood $(\mu \mathrm{g} / \mathrm{ml})$ over time followed single intraperitoneal (ip) injection with $100 \mathrm{mg} / \mathrm{kg} \mathrm{Ag}$ and $\mathrm{ZnO}$ NPs after subtraction of the basal zinc level in the control group, no silver detected in control group . 
Table 1. $\mathrm{Ag}$ and $\mathrm{Zn}$ means concentration $(\mu \mathrm{g} / \mathrm{ml}) \pm \mathrm{ESM}$ in blood over time points after single intraperitoneal injection with $100 \mathrm{mg} / \mathrm{kg} \mathrm{Ag}$ and $\mathrm{ZnO} \mathrm{NPs}$

\begin{tabular}{|c|c|c|c|c|c|c|c|}
\hline \multirow{3}{*}{$\begin{array}{l}\text { Time } \\
5 \mathrm{~min}\end{array}$} & \multicolumn{3}{|c|}{ Ag NPs experimental group } & \multirow{3}{*}{$\begin{array}{c}\text { ZnO NPs } \\
\text { Control } \\
5.00 \pm 0.00\end{array}$} & \multirow{2}{*}{\multicolumn{2}{|c|}{ 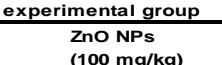 }} & \\
\hline & Control & \multicolumn{2}{|l|}{$\begin{array}{c}\text { Ag NPs } \\
(100 \mathrm{mg} / \mathrm{kg})\end{array}$} & & & & \\
\hline & $0.00 \pm 0.00$ & $15.00 \pm 0.00$ & $\bar{e}$ & & $\bar{a}$ & $28.75 \pm 1.53$ & \\
\hline $10 \mathrm{~min}$ & $0.00 \pm 0.00$ & $21.25 \pm 1.02$ & $\mathrm{t}$ & $5.00 \pm 0.00$ & a & $32.50 \pm 1.02$ & h \\
\hline $15 \mathrm{~min}$ & $0.00 \pm 0.00$ & $25.00 \pm 0.00$ & g & $5.00 \pm 0.51$ & a & $50.63 \pm 0.51$ & 1 \\
\hline $30 \mathrm{~min}$ & $0.00 \pm 0.00$ & $28.75 \pm 1.02$ & h & $5.31 \pm 0.26$ & a & $95.00 \pm 0.00$ & k \\
\hline $60 \mathrm{~min}$ & $0.00 \pm 0.00$ & $57.50 \pm 2.04$ & $\mathrm{j}$ & $5.31 \pm 0.26$ & a & $7.50 \pm 0.00$ & b \\
\hline $2 \mathrm{~h}$ & $0.00 \pm 0.00$ & $7.88 \pm 0.31$ & d & $5.31 \pm 0.26$ & a & $31.25 \pm 0.00$ & $\mathrm{~g}$ \\
\hline $3 \mathbf{h}$ & $0.00 \pm 0.00$ & $3.00 \pm 0.00$ & bc & $5.00 \pm 0.00$ & a & $51.25 \pm 0.00$ & 1 \\
\hline $4 \mathrm{~h}$ & $0.00 \pm 0.00$ & $4.63 \pm 0.31$ & c & $5.00 \pm 0.00$ & a & $37.19 \pm 0.26$ & 1 \\
\hline $6 \mathrm{~h}$ & $0.00 \pm 0.00$ & $22.50 \pm 2.04$ & $\mathrm{t}$ & $5.00 \pm 0.51$ & a & $3.75 \pm 0.51$ & a \\
\hline 1d & $0.00 \pm 0.00$ & $37.50 \pm 2.04$ & $i$ & $5.00 \pm 0.00$ & a & $20.63 \pm 0.51$ & e \\
\hline $2 \mathrm{~d}$ & $0.00 \pm 0.00$ & $1.00 \pm 0.03$ & a & $5.00 \pm 0.51$ & a & $13.44 \pm 0.51$ & d \\
\hline $4 \mathrm{~d}$ & $0.00 \pm 0.00$ & $1.46 \pm 0.03$ & a b & $5.00 \pm 0.00$ & a & $10.94 \pm 0.26$ & c \\
\hline $7 d$ & $0.00 \pm 0.00$ & $1.13 \pm 0.03$ & $a b$ & $5.31 \pm 0.26$ & a & $10.63 \pm 0.00$ & c \\
\hline $9 d$ & $0.00 \pm 0.00$ & $0.54 \pm 0.03$ & a & $5.00 \pm 0.00$ & a & $10.63 \pm 0.26$ & c \\
\hline $11 \mathrm{~d}$ & $0.00 \pm 0.00$ & $0.38 \pm 0.01$ & a & $5.00 \pm 0.00$ & a & $7.81 \pm 0.26$ & b \\
\hline $14 d$ & $0.00 \pm 0.00$ & $0.38 \pm 0.01$ & a & $5.00 \pm 0.00$ & $\mathbf{a}$ & $7.81 \pm 0.26$ & b \\
\hline $18 \mathrm{~d}$ & $0.00 \pm 0.00$ & $0.38 \pm 0.01$ & a & $5.00 \pm 0.00$ & a & $7.81 \pm 0.26$ & b \\
\hline LSD & & 1.90 & & 0.53 & & 1.03 & \\
\hline
\end{tabular}

Table 2. Ag and ZnO NPs blood kinetic parameters means \pm ESM after single intraperitoneal injection

with $100 \mathrm{mg} / \mathrm{kg} \mathrm{Ag}$ and $\mathrm{ZnO} \mathrm{NPs}$

\begin{tabular}{|c|c|c|c|c|c|c|c|c|}
\hline & $\begin{array}{l}\text { dose } \\
\mathrm{mg} / \mathrm{kg}\end{array}$ & $\begin{array}{l}\text { CMax. } \\
\mu \mathrm{g} / \mathrm{ml}\end{array}$ & $\begin{array}{l}\text { TMax. } \\
\min .\end{array}$ & $\begin{array}{l}T 1 / 2 \text { Abs. } \\
\text { min. }\end{array}$ & $\begin{array}{l}\text { Last T1/2 Elem. } \\
d\end{array}$ & $\begin{array}{l}\text { Clerance } \\
1 / \text { kg.d }\end{array}$ & $\begin{array}{l}k \text { Elem. } \\
1 / d\end{array}$ & $\begin{array}{l}\text { Apparent Vd } \\
\text { l.kg }\end{array}$ \\
\hline Ag NPs & 100 & $57.50 \pm 2.50$ & $60.00 \pm 0.00$ & $26.32 \pm 0.57$ & $8.39 \pm 0.23$ & $0.43 \pm 0.03$ & $0.08 \pm 0.00$ & $5.16 \pm 0.25$ \\
\hline ZnONPs & 100 & $95.0 \pm 0.00$ & $30.00 \pm 0.00$ & $14.30 \pm 0.00$ & $2.38 \pm 0.08$ & $0.87 \pm 0.03$ & $0.29 \pm 0.00$ & $2.99 \pm 0.02$ \\
\hline
\end{tabular}

\subsection{Ag and ZnO NPs distribution}

Regardless the route of administration, nanoparticles may become available systematically and start potential interaction with different portions in plasma, blood contents, and coagulation factors [30], this special interaction have important influence on further steps of distribution addition to nano excretion [31]. Figurs 3 and 4 ,Tables 3 and 4 showed the distribution profile of $\mathrm{Ag}$ and $\mathrm{ZnO}$ NPs after single intraperitoneal injected dose of $100 \mathrm{mg} / \mathrm{Kg}$. With an overview, Ag and $\mathrm{Zn}$ were distributed to all studied organs. The highest levels of silver and zinc showed after 60 and 30 min of injection respectively with the same manner with their levels in blood depending in their speed on some factors starting from the half life of absorption which illustrated by increasing retention time of Ag NPs in peritoneal cavity compared with ZnO NPs by the effect of high molecular weight as discussed before and the amount of ions released from each kind of nnanoparticles [26]. At time period between $1 \mathrm{~h}$ to 1 day the translocation appeared to began in different studded organs which may referred to the redistribution of monocytes with remaining in spleen as a reservoir [32] where silver and zinc in particulate, ionic, or complex binding forms may be trans located from the initial uptake tissues to other parts of the body through the circulatory system [33]-[36]. After time point of 1-2 days gradual dropping were became obvious for both of $\mathrm{Ag}$ and $\mathrm{Zn}$ concentrations in various studied organs with faster rate for $\mathrm{Zn}$ as larger clearance rate which recorded for $\mathrm{ZnO}$ NPs in blood. Equilibrium become clear after 7 days for $\mathrm{Ag}$ and not quite cleared for $\mathrm{Zn}$ concentrations .

Liver and spleen which have the dominant role in immune system [37], hold more nanoparticles pending systematic inflammation that led to shorthand retention of nanoparticles by other organs [38], and their clearing role by the interaction of itself protein effectively with nanoparticles with altering the antigenicity and inducing the autoimmune responses, resulting complex of nanoparticle- protein that can facilitate dendritic cells antigen up taking [39]. But as effect of masses different between liver and spleen which have smaller one [40] , making spleen have grater accumulation per unit of mass specially during the first hour after injection . Interesting high concentrations of $\mathrm{Zn}$ were observed in intestine specially at early time of injection (10 min-1 h) which may be related to the direct absorption of ZnO NPs that resides initially in the visceral tissues surrounding area from intrperitoneal cavity to intestine [41], in addition to excreted nanoparticles 
from the liver via the biliary pathway [42]. In lung a considerable concentration of $\mathrm{Ag}$ and $\mathrm{Zn}$ were noted in this study which confirmed the translocation [43], also redistribution of nanoparticles over time which indicated before by Dziendzikowska [44] using TEM in rat alveolar macrophages after 7 days of intravenous injection with $5 \mathrm{mg} / \mathrm{kg} \mathrm{Ag} \mathrm{NPs}$. The noted concentrations decreased over time to record their lowest levels after 9 days and 11 days for $\mathrm{Ag}$ and $\mathrm{Zn}$ respectively. This study remarked a considerable $\mathrm{Ag}$ and $\mathrm{Zn}$ levels in brain with $2.26 \pm 0.00 \mu \mathrm{g} / \mathrm{gm}$ and $32.81 \pm 0.31 \mu \mathrm{g} / \mathrm{gm}$ respectively, these results corroborate the ability of both types of nanoparticles to cross the blood brain barrier (BBB) as proved in previous studies of [45],[16],[46],[44],[47]. The equilibrium were not well clarified at the end time points specially between 7-18 days as some rising in $\mathrm{Ag}$ and $\mathrm{Zn}$ concentrations in brain which may related to improved absorption by endothelial cells and as a result facilitate their uptake and translocation through blood vessels wall by process of transcytosis [48]. These results came with those of [44] in increasing silver concentration in brain at the end point $28 \mathrm{~d}$ of their experiment after single intravenous injection with $5 \mathrm{mg} / \mathrm{kg}$ of 20 and $200 \mathrm{~nm} \mathrm{Ag} \mathrm{NPs}$. There is an essential evidence proposing that Ag NPs can pass the blood testis barrier BTB as the occurrence of silver in the tissue of testicles which confirmed by exposing animals to Ag NPs through different routs of administration [36],[49],[50], in current study the maximum concentrations of $\mathrm{Ag}$ and $\mathrm{ZnO}$ were $9.57 \pm 0.43$ and $48.45 \pm 0.39 \mu \mathrm{g} / \mathrm{gm}$ after 60 and $30 \mathrm{~min}$ of injection respectively with slow rate of elimination over experimental time points.

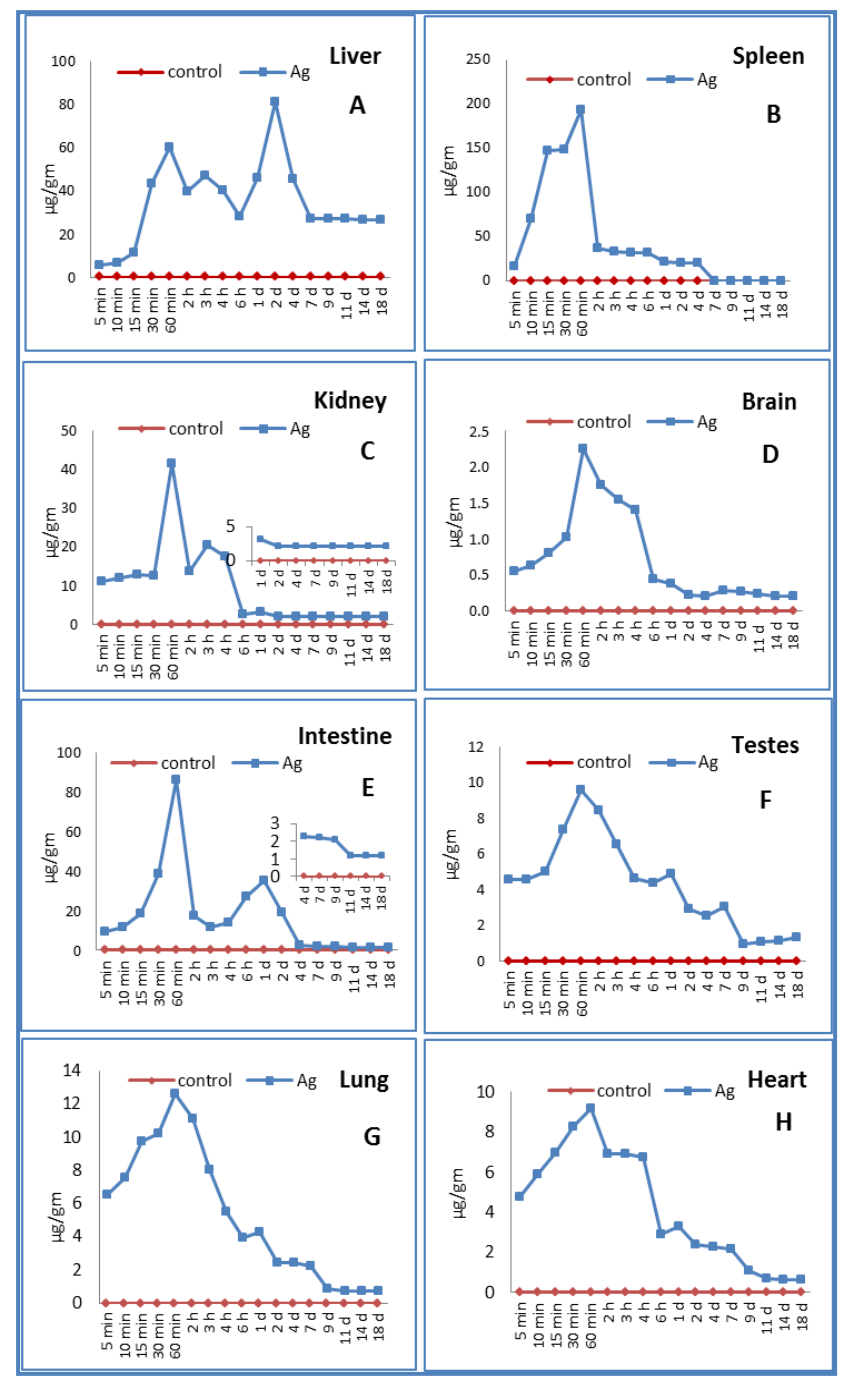

Figure 3. Silver concentrations means $(\mu \mathrm{g} / \mathrm{gm})$ in (A) Liver, (B) Spleen, (C) Kidney , (D) Brain, (E) Intestine, (F) Testes, (G) Lung, and (H) Heart over time following single intraperitoneal injection with $100 \mathrm{mg} / \mathrm{kg}$ Ag NPs.

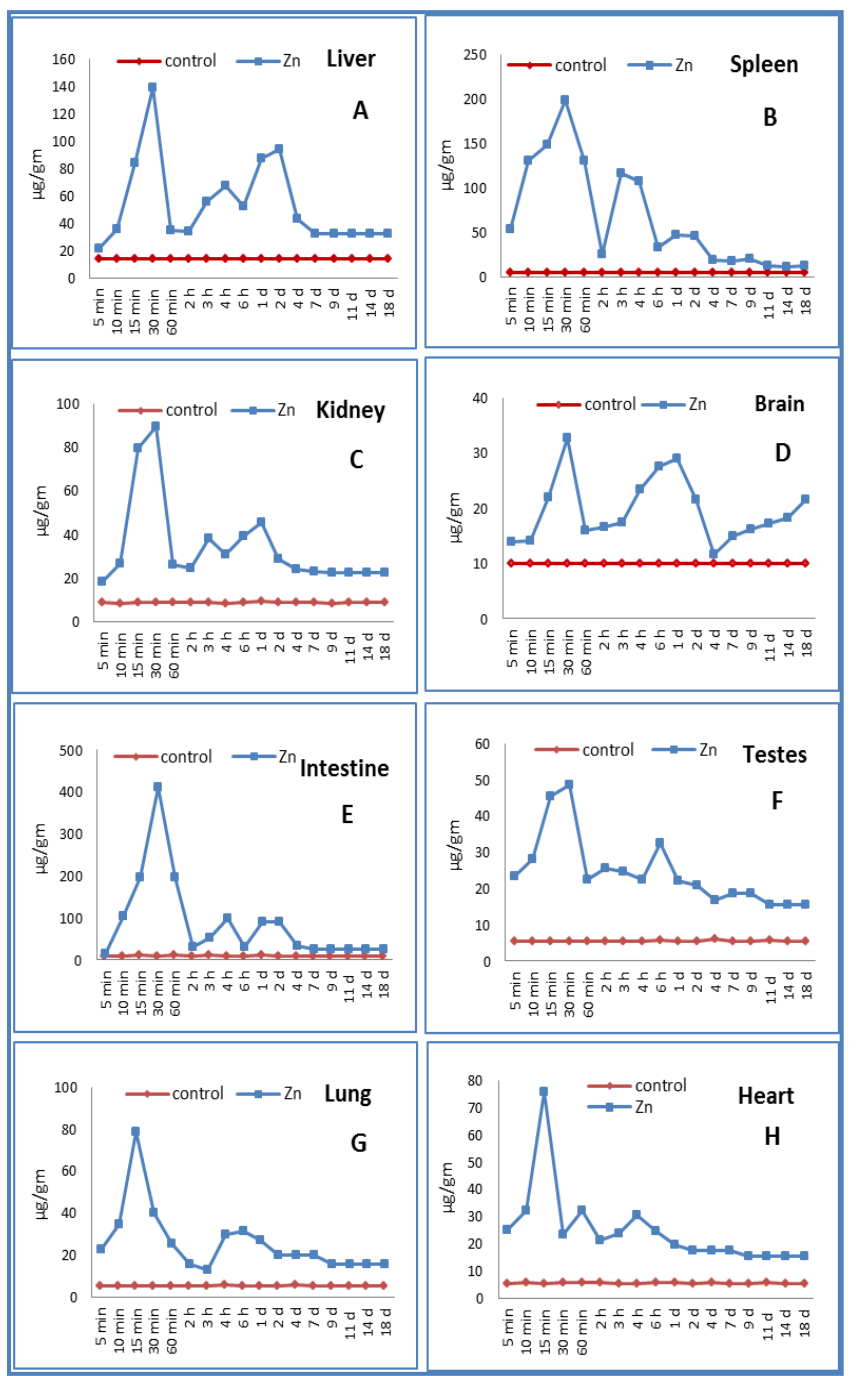

Figure 4. Zinc concentrations means $(\mu \mathrm{g} / \mathrm{gm})$ in $(\mathrm{A})$ Liver, (B)Spleen, (C)Kidney, (D) Brain, (E) Intestine, (F) Testes, (G) Lung, and (H) Heart over time following single intraperitoneal injection with 100 $\mathrm{mg} / \mathrm{kg} \mathrm{ZnO} \mathrm{NPs.}$ 
Table 3. Silver concentrations means $(\mu \mathrm{g} / \mathrm{gm}) \pm$ SEM in liver, spleen, kidney, brain, intestine, testes, lung, heart over experimental time points following single intraperitoneal injection with $100 \mathrm{mg} / \mathrm{kg} \mathrm{Ag} \mathrm{NPs}$.

\begin{tabular}{|c|c|c|c|c|c|c|c|c|c|c|c|c|}
\hline \multirow[t]{2}{*}{ Time } & \multicolumn{2}{|c|}{ Liver } & & \multicolumn{2}{|c|}{ Spleen } & & \multicolumn{2}{|c|}{ Kidney } & & \multicolumn{2}{|c|}{ Brain } & \\
\hline & Control & $\begin{array}{c}\text { Ag NPs } \\
(100 \mathrm{mg} / \mathrm{kg})\end{array}$ & & Control & $\begin{array}{l}\text { Ag NPs } \\
(100 \mathrm{mg} / \mathrm{kg})\end{array}$ & & Control & $\begin{array}{c}\text { Ag NPs } \\
(100 \mathrm{mg} / \mathrm{kg})\end{array}$ & & Control & $\begin{array}{l}\text { Ag NPs } \\
(100 \mathrm{mg} / \mathrm{kg})\end{array}$ & \\
\hline $5 \mathrm{~min}$ & $0.00 \pm 0.00$ & $6.00 \pm 0.00$ & $\mathrm{a}$ & $0.00 \pm 0.00$ & $15.74 \pm 0.93$ & b & $0.00 \pm 0.00$ & $11.09 \pm 0.22$ & c & $0.00 \pm 0.00$ & $0.54 \pm 0.07$ & \\
\hline $10 \mathrm{~min}$ & $0.00 \pm 0.00$ & $6.75 \pm 0.25$ & a & $0.00 \pm 0.00$ & $69.44 \pm 0.93$ & h & $0.00 \pm 0.00$ & $11.96 \pm 0.22$ & d & $0.00 \pm 0.00$ & $0.63 \pm 0.02$ & e \\
\hline $15 \min$ & $0.00 \pm 0.00$ & $11.75 \pm 0.25$ & b & $0.00 \pm 0.00$ & $147.22 \pm 0.93$ & & $0.00 \pm 0.00$ & $12.83 \pm 0.22$ & 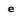 & $0.00 \pm 0.00$ & $0.80 \pm 0.02$ & \\
\hline $30 \mathrm{~min}$ & $0.00 \pm 0.00$ & $43.52 \pm 0.93$ & ef & $0.00 \pm 0.00$ & $148.15 \pm 1.85$ & i & $0.00 \pm 0.00$ & $12.61 \pm 0.43$ & d & $0.00 \pm 0.00$ & $1.02 \pm 0.07$ & g \\
\hline $60 \mathrm{~min}$ & $0.00 \pm 0.00$ & $60.19 \pm 0.93$ & h & $0.00 \pm 0.00$ & $193.52 \pm 0.93$ & j & $0.00 \pm 0.00$ & $41.67 \pm 0.93$ & $i$ & $0.00 \pm 0.00$ & $2.26 \pm 0.00$ & k \\
\hline $2 \mathrm{~h}$ & $0.00 \pm 0.00$ & $39.81 \pm 0.93$ & d & $0.00 \pm 0.00$ & $36.11 \pm 0.93$ & $\mathrm{~g}$ & $0.00 \pm 0.00$ & $13.89 \pm 0.93$ & 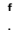 & $0.00 \pm 0.00$ & $1.75 \pm 0.05$ & j \\
\hline $3 \mathrm{~h}$ & $0.00 \pm 0.00$ & $47.22 \pm 0.93$ & g & $0.00 \pm 0.00$ & $33.33 \pm 1.85$ & $\mathrm{f}$ & $0.00 \pm 0.00$ & $20.50 \pm 0.50$ & $\mathrm{~h}$ & $0.00 \pm 0.00$ & $1.55 \pm 0.05$ & \\
\hline $4 \mathrm{~h}$ & $0.00 \pm 0.00$ & $40.74 \pm 1.85$ & de & $0.00 \pm 0.00$ & $31.48 \pm 0.00$ & e & $0.00 \pm 0.00$ & $17.50 \pm 0.50$ & g & $0.00 \pm 0.00$ & $1.40 \pm 0.10$ & $\mathrm{~h}$ \\
\hline $6 \mathrm{~h}$ & $0.00 \pm 0.00$ & $28.70 \pm 0.93$ & c & $0.00 \pm 0.00$ & $31.48 \pm 1.85$ & 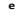 & $0.00 \pm 0.00$ & $2.57 \pm 0.00$ & ab & $0.00 \pm 0.00$ & $0.44 \pm 0.04$ & 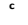 \\
\hline 1d & $0.00 \pm 0.00$ & $46.43 \pm 1.19$ & $\mathrm{fg}$ & $0.00 \pm 0.00$ & $21.30 \pm 0.93$ & d & $0.00 \pm 0.00$ & $3.14 \pm 0.14$ & b & $0.00 \pm 0.00$ & $0.38 \pm 0.05$ & c \\
\hline $2 \mathrm{~d}$ & $0.00 \pm 0.00$ & $81.25 \pm 2.08$ & i & $0.00 \pm 0.00$ & $19.44 \pm 0.93$ & c & $0.00 \pm 0.00$ & $2.21 \pm 0.07$ & a & $0.00 \pm 0.00$ & $0.22 \pm 0.02$ & $\mathrm{a}$ \\
\hline $4 \mathrm{~d}$ & $0.00 \pm 0.00$ & $45.83 \pm 4.17$ & t & $0.00 \pm 0.00$ & $19.44 \pm 0.93$ & c & $0.00 \pm 0.00$ & $2.14 \pm 0.14$ & a & $0.00 \pm 0.00$ & $0.20 \pm 0.01$ & a \\
\hline $7 \mathrm{~d}$ & $0.00 \pm 0.00$ & $27.50 \pm 2.50$ & 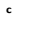 & $0.00 \pm 0.00$ & $0.07 \pm 0.00$ & a & $0.00 \pm 0.00$ & $2.21 \pm 0.07$ & a & $0.00 \pm 0.00$ & $0.28 \pm 0.01$ & b \\
\hline $9 d$ & $0.00 \pm 0.00$ & $27.50 \pm 1.67$ & c & $0.00 \pm 0.00$ & $0.07 \pm 0.00$ & a & $0.00 \pm 0.00$ & $2.21 \pm 0.07$ & a & $0.00 \pm 0.00$ & $0.26 \pm 0.01$ & ab \\
\hline $11 \mathrm{~d}$ & $0.00 \pm 0.00$ & $27.29 \pm 2.29$ & c & $0.00 \pm 0.00$ & $0.06 \pm 0.00$ & a & $0.00 \pm 0.00$ & $2.21 \pm 0.07$ & a & $0.00 \pm 0.00$ & $0.23 \pm 0.01$ & a \\
\hline $14 \mathrm{~d}$ & $0.00 \pm 0.00$ & $27.08 \pm 2.08$ & c & $0.00 \pm 0.00$ & $0.06 \pm 0.00$ & a & $0.00 \pm 0.00$ & $2.21 \pm 0.07$ & a & $0.00 \pm 0.00$ & $0.21 \pm 0.01$ & a \\
\hline $18 \mathrm{~d}$ & $0.00 \pm 0.00$ & $27.08 \pm 2.08$ & c & $0.00 \pm 0.00$ & $0.06 \pm 0.00$ & a & $0.00 \pm 0.00$ & $2.21 \pm 0.07$ & a & $0.00 \pm 0.00$ & $0.21 \pm 0.01$ & a \\
\hline LSD & No & 3.00 & & No & 1.70 & & No & 0.70 & & No & 0.07 & \\
\hline \multirow[t]{3}{*}{ Time } & \multicolumn{2}{|c|}{ Small intestine } & & \multicolumn{3}{|c|}{ Testes } & \multicolumn{3}{|c|}{ Lung } & \multicolumn{3}{|c|}{ Heart } \\
\hline & \multicolumn{3}{|c|}{ Ag NPs } & \multicolumn{3}{|c|}{ Ag NPs } & \multicolumn{3}{|c|}{ Ag NPs } & \multicolumn{3}{|c|}{ Ag NPs } \\
\hline & Control & $(100 \mathrm{mg} / \mathrm{kg})$ & & Control & $(100 \mathrm{mg} / \mathrm{kg})$ & & Control & $(100 \mathrm{mg} / \mathrm{kg})$ & & Control & $(100 \mathrm{mg} / \mathrm{kg})$ & \\
\hline $5 \min$ & $0.00 \pm 0.00$ & $9.25 \pm 0.75$ & b & $0.00 \pm 0.00$ & $4.57 \pm 0.22$ & $\overline{\mathrm{a}}$ & $0.00 \pm 0.00$ & $6.52 \pm 0.43$ & $\bar{e}$ & $0.00 \pm 0.00$ & $4.78 \pm 0.00$ & \\
\hline $10 \mathrm{~min}$ & $0.00 \pm 0.00$ & $11.50 \pm 0.50$ & c & $0.00 \pm 0.00$ & $4.57 \pm 0.22$ & $c d$ & $0.00 \pm 0.00$ & $7.61 \pm 0.22$ & f & $0.00 \pm 0.00$ & $5.87 \pm 0.22$ & g \\
\hline $15 \min$ & $0.00 \pm 0.00$ & $18.75 \pm 0.75$ & e & $0.00 \pm 0.00$ & $5.00 \pm 0.22$ & d & $0.00 \pm 0.00$ & $9.78 \pm 0.22$ & $\mathrm{~h}$ & $0.00 \pm 0.00$ & $6.96 \pm 0.00$ & $\mathrm{~h}$ \\
\hline $30 \mathrm{~min}$ & $0.00 \pm 0.00$ & $38.89 \pm 1.85$ & $\mathrm{~h}$ & $0.00 \pm 0.00$ & $7.39 \pm 0.43$ & $f$ & $0.00 \pm 0.00$ & $10.22 \pm 0.65$ & $\mathrm{~h}$ & $0.00 \pm 0.00$ & $8.26 \pm 0.00$ & i \\
\hline $60 \mathrm{~min}$ & $0.00 \pm 0.00$ & $86.11 \pm 0.93$ & i & $0.00 \pm 0.00$ & $9.57 \pm 0.43$ & $\mathrm{~h}$ & $0.00 \pm 0.00$ & $12.61 \pm 0.00$ & j & $0.00 \pm 0.00$ & $9.15 \pm 0.02$ & j \\
\hline $2 \mathrm{~h}$ & $0.00 \pm 0.00$ & $17.50 \pm 0.50$ & e & $0.00 \pm 0.00$ & $8.46 \pm 0.77$ & g & $0.00 \pm 0.00$ & $11.15 \pm 0.38$ & & $0.00 \pm 0.00$ & $6.92 \pm 0.00$ & $\mathrm{~h}$ \\
\hline $3 \mathrm{~h}$ & $0.00 \pm 0.00$ & $11.50 \pm 0.50$ & c & $0.00 \pm 0.00$ & $6.54 \pm 0.38$ & e & $0.00 \pm 0.00$ & $8.08 \pm 0.38$ & g & $0.00 \pm 0.00$ & $6.92 \pm 0.00$ & $\mathrm{~h}$ \\
\hline $4 \mathrm{~h}$ & $0.00 \pm 0.00$ & $14.00 \pm 0.00$ & d & $0.00 \pm 0.00$ & $4.62 \pm 0.38$ & cd & $0.00 \pm 0.00$ & $5.50 \pm 0.00$ & d & $0.00 \pm 0.00$ & $6.75 \pm 0.75$ & $\mathrm{~h}$ \\
\hline $6 \mathrm{~h}$ & $0.00 \pm 0.00$ & $27.00 \pm 3.00$ & f & $0.00 \pm 0.00$ & $4.38 \pm 0.38$ & c & $0.00 \pm 0.00$ & $3.95 \pm 0.47$ & c & $0.00 \pm 0.00$ & $2.91 \pm 0.12$ & d \\
\hline $1 d$ & $0.00 \pm 0.00$ & $35.00 \pm 3.00$ & g & $0.00 \pm 0.00$ & $4.88 \pm 0.38$ & cd & $0.00 \pm 0.00$ & $4.30 \pm 0.35$ & c & $0.00 \pm 0.00$ & $3.26 \pm 0.23$ & e \\
\hline $2 \mathrm{~d}$ & $0.00 \pm 0.00$ & $19.00 \pm 1.00$ & e & $0.00 \pm 0.00$ & $2.93 \pm 0.07$ & b & $0.00 \pm 0.00$ & $2.43 \pm 0.00$ & b & $0.00 \pm 0.00$ & $2.36 \pm 0.07$ & c \\
\hline $4 \mathrm{~d}$ & $0.00 \pm 0.00$ & $2.29 \pm 0.14$ & a & $0.00 \pm 0.00$ & $2.57 \pm 0.14$ & b & $0.00 \pm 0.00$ & $2.43 \pm 0.14$ & b & $0.00 \pm 0.00$ & $2.29 \pm 0.00$ & c \\
\hline $7 \mathrm{~d}$ & $0.00 \pm 0.00$ & $2.21 \pm 0.07$ & a & $0.00 \pm 0.00$ & $3.07 \pm 0.07$ & b & $0.00 \pm 0.00$ & $2.21 \pm 0.07$ & b & $0.00 \pm 0.00$ & $2.14 \pm 0.00$ & c \\
\hline $9 \mathrm{~d}$ & $0.00 \pm 0.00$ & $2.07 \pm 0.07$ & a & $0.00 \pm 0.00$ & $0.97 \pm 0.03$ & a & $0.00 \pm 0.00$ & $0.89 \pm 0.04$ & a & $0.00 \pm 0.00$ & $1.07 \pm 0.07$ & b \\
\hline $11 \mathrm{~d}$ & $0.00 \pm 0.00$ & $1.21 \pm 0.07$ & $a$ & $0.00 \pm 0.00$ & $1.07 \pm 0.07$ & a & $0.00 \pm 0.00$ & $0.75 \pm 0.04$ & a & $0.00 \pm 0.00$ & $0.68 \pm 0.04$ & a \\
\hline $14 \mathrm{~d}$ & $0.00 \pm 0.00$ & $1.21 \pm 0.07$ & a & $0.00 \pm 0.00$ & $1.17 \pm 0.03$ & a & $0.00 \pm 0.00$ & $0.75 \pm 0.04$ & a & $0.00 \pm 0.00$ & $0.61 \pm 0.04$ & a \\
\hline $\begin{array}{l}18 \mathrm{~d} \\
\text { LSD }\end{array}$ & $\begin{array}{l}0.00 \pm 0.00 \\
\text { No }\end{array}$ & $\begin{array}{l}1.21 \pm 0.07 \\
2.03\end{array}$ & a & $\begin{array}{l}0.00 \pm 0.00 \\
\text { No }\end{array}$ & $\begin{array}{l}1.37 \pm 0.03 \\
0.53\end{array}$ & a & $\begin{array}{l}0.00 \pm 0.00 \\
\text { No }\end{array}$ & $\begin{array}{l}0.75 \pm 0.04 \\
0.47\end{array}$ & a & $\begin{array}{l}0.00 \pm 0.00 \\
\text { No }\end{array}$ & $\begin{array}{l}0.64 \pm 0.07 \\
0.34\end{array}$ & a \\
\hline
\end{tabular}

Table 4. Zinc concentrations means $(\mu \mathrm{g} / \mathrm{gm}) \pm \mathrm{SEM}$ in liver, spleen, kidney, brain, intestine, testes, lung and heart over experimental time points following single intraperitoneal injection with $100 \mathrm{mg} / \mathrm{kg} \mathrm{Ag} \mathrm{NPs}$.

\begin{tabular}{|c|c|c|c|c|c|c|c|c|c|c|c|c|}
\hline \multirow[t]{2}{*}{ Time } & \multicolumn{2}{|c|}{ Liver } & & \multicolumn{2}{|c|}{ Spleen } & & \multicolumn{2}{|c|}{ Kidney } & & \multicolumn{3}{|c|}{ Brain } \\
\hline & Control & $\begin{array}{l}\mathrm{ZnONPs} \\
(100 \mathrm{mg} / \mathrm{kg})\end{array}$ & & Control & $\begin{array}{l}\mathrm{ZnO} N P s \\
(100 \mathrm{mg} / \mathrm{kg})\end{array}$ & & Control & $\begin{array}{c}\text { ZnO NPs } \\
(100 \mathrm{mg} / \mathrm{kg})\end{array}$ & & Control & $\begin{array}{l}\text { ZnO NPs } \\
(100 \mathrm{mg} / \mathrm{kg})\end{array}$ & \\
\hline $5 \min$ & $14.39 \pm 0.24^{a}$ & $22.22 \pm 1.85$ & a & $4.88 \pm 0.49^{a}$ & $52.86 \pm 0.48$ & $\mathrm{~h}$ & $9.02 \pm 0.24^{a}$ & $18.57 \pm 0.48$ & a & $10.00 \pm 0.24^{a}$ & $13.84 \pm 0.45$ & b \\
\hline $10 \mathrm{~min}$ & $14.15 \pm 0.49^{a}$ & $36.19 \pm 0.95$ & $c^{c}$ & $4.63 \pm 0.73$ & $130.95 \pm 2.38$ & k & $8.78 \pm 0.49^{a}$ & $27.14 \pm 0.48$ & & $10.00 \pm 0.24$ & $14.23 \pm 0.38$ & bc \\
\hline $15 \min$ & $14.39 \pm 0.24$ a & $84.29 \pm 0.48$ & $\mathrm{~h}$ & $4.63 \pm 0.24 a$ & $148.57 \pm 0.95$ & 1 & $9.02 \pm 0.24$ a & $79.52 \pm 0.48$ & k & $10.00 \pm 0.24 \mathrm{a}$ & $21.92 \pm 0.38$ & $\mathrm{~g}$ \\
\hline $30 \mathrm{~min}$ & $14.39 \pm 0.24$ a & $139.05 \pm 0.95$ & k & $4.88 \pm 0.49^{a}$ & $198.10 \pm 0.95$ & $\mathrm{~m}$ & $9.27 \pm 0.49^{a}$ & $89.52 \pm 0.95$ & & $10.00 \pm 0.24$ a & $32.81 \pm 0.31$ & k \\
\hline $60 \mathrm{~min}$ & $14.39 \pm 0.24 \mathrm{a}$ & $35.24 \pm 0.95$ & $c^{c}$ & $4.63 \pm 0.73$ & $130.00 \pm 2.38$ & k & $9.02 \pm 0.24^{a}$ & $26.19 \pm 0.48$ & ef & $10.00 \pm 0.24$ & $16.09 \pm 0.43$ & d \\
\hline $2 \mathrm{~h}$ & $14.15 \pm 0.49^{a}$ & $34.35 \pm 0.43$ & bc & $4.63 \pm 0.73^{a}$ & $25.22 \pm 0.00$ & d & $9.27 \pm 0.49^{a}$ & $24.78 \pm 0.43$ & de & $10.00 \pm 0.24^{a}$ & $16.52 \pm 0.87$ & de \\
\hline $3 \mathbf{h}$ & $14.39 \pm 0.24^{a}$ & $56.09 \pm 0.43$ & f & $4.63 \pm 1.22^{\mathrm{a}}$ & $116.52 \pm 0.87$ & j & $9.02 \pm 0.24 \mathrm{a}^{\mathrm{a}}$ & $38.26 \pm 0.00$ & i & $10.00 \pm 0.24$ a & $17.39 \pm 0.00$ & e \\
\hline $4 \mathrm{~h}$ & $14.15 \pm 0.49$ a & $67.83 \pm 0.87$ & g & $4.88 \pm 0.49 \mathrm{a}$ & $106.96 \pm 0.00$ & ' & $8.78 \pm 0.49 \mathrm{a}$ & $30.87 \pm 0.43$ & ${ }^{\mathrm{h}}$ & $10.00 \pm 0.24 \mathrm{a}$ & $23.48 \pm 0.00$ & h \\
\hline $6 \mathrm{~h}$ & $14.15 \pm 0.49$ a & $52.53 \pm 2.02$ & $e^{e}$ & $4.63 \pm 0.73$ & $33.33 \pm 1.01$ & e & $9.02 \pm 0.24 \mathrm{a}^{\mathrm{a}}$ & $39.39 \pm 1.01$ & $i$ & $10.00 \pm 0.24 \mathrm{a}$ & $27.50 \pm 0.50$ & \\
\hline 1d & $14.15 \pm 0.49^{a}$ & $87.88 \pm 1.01$ & i & $4.88 \pm 0.98^{a}$ & $47.47 \pm 1.01$ & $\mathrm{~g}$ & $9.51 \pm 0.24 \mathrm{a}^{\mathrm{a}}$ & $45.96 \pm 1.52$ & j & $10.00 \pm 0.24 \mathrm{a}$ & $29.00 \pm 1.00$ & j \\
\hline $2 \mathrm{~d}$ & $14.39 \pm 0.24^{a}$ & $93.89 \pm 0.56$ & j & $4.63 \pm 0.24 \mathrm{a}$ & $45.70 \pm 0.54$ & $\mathrm{f}$ & $9.02 \pm 0.24 \mathrm{a}$ & $29.03 \pm 2.15$ & $\mathrm{~g}$ & $10.00 \pm 0.24^{a}$ & $21.67 \pm 0.56$ & $\mathrm{~g}$ \\
\hline $4 \mathrm{~d}$ & $14.39 \pm 0.24{ }^{a}$ & $43.33 \pm 4.44$ & d & $4.63 \pm 0.73^{a}$ & $18.82 \pm 0.54$ & bc & $9.27 \pm 0.49 \mathrm{a}$ & $24.19 \pm 0.54$ & ${ }^{c d}$ & $10.00 \pm 0.24 \mathrm{a}$ & $11.67 \pm 0.56$ & a \\
\hline $7 d$ & $14.15 \pm 0.49^{a}$ & $32.78 \pm 0.56$ & b & $4.63 \pm 0.24^{a}$ & $17.74 \pm 0.54$ & b & $9.02 \pm 0.24^{a}$ & $23.12 \pm 0.54$ & bc & $10.00 \pm 0.24$ a & $15.00 \pm 0.56$ & \\
\hline $9 d$ & $14.39 \pm 0.24$ a & $32.78 \pm 0.56$ & b & $4.88 \pm 0.98^{a}$ & $19.89 \pm 0.54$ & c & $8.78 \pm 0.49 \mathrm{a}$ & $22.58 \pm 0.00$ & b & $10.00 \pm 0.24 \mathrm{a}$ & $16.11 \pm 0.56$ & d \\
\hline $11 \mathrm{~d}$ & $14.15 \pm 0.49^{a}$ & $32.78 \pm 0.56$ & b & $4.88 \pm 0.49^{a}$ & $12.37 \pm 0.54$ & a & $9.02 \pm 0.24 \mathrm{a}$ & $22.58 \pm 1.08$ & b & $10.00 \pm 0.24^{a}$ & $17.22 \pm 0.56$ & e \\
\hline $14 \mathrm{~d}$ & $14.39 \pm 0.24$ a & $32.78 \pm 0.56$ & b & $4.63 \pm 0.73^{a}$ & $11.29 \pm 0.54$ & a & $9.02 \pm 0.24 \mathrm{a}$ & $22.58 \pm 1.08$ & b & $10.00 \pm 0.24 \mathrm{a}$ & $18.33 \pm 0.56$ & \\
\hline $18 \mathrm{~d}$ & $14.39 \pm 0.24 \mathrm{a}$ & $32.78 \pm 0.56$ & b & $4.88 \pm 0.49 \mathrm{a}$ & $11.83 \pm 0.00$ & a & $9.02 \pm 0.24 \mathrm{a}$ & $22.58 \pm 0.00$ & b & $10.00 \pm 0.24 \mathrm{a}$ & $21.67 \pm 0.56$ & $\mathrm{~g}$ \\
\hline LSD & 0.61 & 2.36 & & 1.10 & 1.71 & & 0.81 & 1.46 & & 0.40 & 0.90 & \\
\hline \multirow[t]{3}{*}{ Time } & \multicolumn{3}{|c|}{ Small intestine } & \multicolumn{3}{|c|}{ Testes } & \multicolumn{3}{|c|}{ Lung } & \multicolumn{3}{|c|}{ Heart } \\
\hline & \multicolumn{3}{|c|}{ ZnONPs } & \multicolumn{3}{|c|}{ ZnO NPs } & \multicolumn{3}{|c|}{ Zno NPs } & \multicolumn{3}{|c|}{ ZnO NPs } \\
\hline & Control & $(100 \mathrm{mg} / \mathrm{kg})$ & & Control & $(100 \mathrm{mg} / \mathrm{kg})$ & & Control & $(100 \mathrm{mg} / \mathrm{kg})$ & & Control & $(100 \mathrm{mg} / \mathrm{kg})$ & \\
\hline $5 \min$ & $10.73 \pm 0.00^{a}$ & $15.24 \pm 0.95$ & a & $5.61 \pm 0.24^{\mathrm{a}}$ & $23.33 \pm 0.48$ & e & $5.12 \pm 0.24^{a}$ & $22.77 \pm 0.45$ & d & $5.61 \pm 0.24^{a}$ & $25.00 \pm 0.00$ & g \\
\hline $10 \mathrm{~min}$ & $10.24 \pm 0.49$ a & $103.81 \pm 0.00$ & $\mathrm{~h}$ & $5.37 \pm 0.49^{a}$ & $28.13 \pm 0.45$ & g & $5.37 \pm 0.49 \mathrm{a}$ & $34.76 \pm 0.48$ & i & $5.85 \pm 0.49^{a}$ & $32.38 \pm 0.95$ & \\
\hline $15 \mathrm{~min}$ & $10.85 \pm 0.37$ a & $196.19 \pm 0.95$ & & $5.61 \pm 0.24^{a}$ & $45.34 \pm 0.62$ & i & $5.61 \pm 0.24 \mathrm{a}^{\mathrm{a}}$ & $78.68 \pm 1.16$ & k & $5.61 \pm 0.24$ a & $75.58 \pm 0.39$ & $\mathrm{j}$ \\
\hline $30 \mathrm{~min}$ & $10.73 \pm 0.49^{a}$ & $411.90 \pm 0.48$ & 1 & $5.61 \pm 0.73^{a}$ & $48.45 \pm 0.39$ & j & $5.12 \pm 0.24 \mathrm{a}^{\mathrm{a}}$ & $40.06 \pm 0.31$ & j & $5.85 \pm 0.49^{a}$ & $23.29 \pm 0.93$ & e \\
\hline $60 \mathrm{~min}$ & $10.85 \pm 0.12 \mathrm{a}$ & $196.19 \pm 0.95$ & & $5.61 \pm 0.24 \mathrm{a}$ & $22.38 \pm 0.48$ & d & $5.37 \pm 0.49 \mathrm{a}$ & $25.24 \pm 0.48$ & e & $6.10 \pm 0.24$ a & $32.38 \pm 0.95$ & \\
\hline $2 \mathrm{~h}$ & $10.49 \pm 0.24$ a & $31.30 \pm 0.87$ & c & $5.61 \pm 0.73^{a}$ & $25.65 \pm 0.43$ & $f$ & $5.61 \pm 0.24 \mathrm{a}^{\mathrm{a}}$ & $15.65 \pm 0.00$ & b & $6.10 \pm 0.24 \mathrm{a}$ & $21.30 \pm 0.43$ & d \\
\hline $3 \mathbf{h}$ & $10.98 \pm 0.24$ a & $53.48 \pm 0.43$ & e & $5.61 \pm 0.24 \mathrm{a}$ & $24.78 \pm 0.43$ & f & $5.12 \pm 0.24 \mathrm{a}$ & $13.04 \pm 0.87$ & a & $5.61 \pm 0.73^{a}$ & $23.91 \pm 0.43$ & 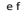 \\
\hline $4 \mathrm{~h}$ & $10.73 \pm 0.49^{a}$ & $99.57 \pm 0.43$ & $\mathrm{~g}$ & $5.37 \pm 0.49^{a}$ & $22.61 \pm 0.00$ & de & $5.85 \pm 0.49 a^{a}$ & $30.00 \pm 0.43$ & $\mathrm{~g}$ & $5.61 \pm 0.24^{a}$ & $30.43 \pm 0.00$ & \\
\hline $6 \mathrm{~h}$ & $10.24 \pm 0.49$ a & $32.32 \pm 5.05$ & $c$ & $5.85 \pm 0.49 a$ & $32.50 \pm 0.50$ & $\mathrm{~h}$ & $5.61 \pm 0.24 \mathrm{a}$ & $31.50 \pm 1.50$ & $\mathrm{~h}$ & $5.85 \pm 0.49 a$ & $24.50 \pm 0.50$ & $\mathrm{fg}$ \\
\hline 1d & $10.85 \pm 0.85^{a}$ & $90.40 \pm 0.51$ & & $5.37 \pm 0.49^{a}$ & $22.00 \pm 2.00$ & cd & $5.12 \pm 0.24^{a}$ & $27.00 \pm 1.00$ & f & $5.85 \pm 0.49$ a & $19.50 \pm 0.50$ & $c$ \\
\hline $2 \mathrm{~d}$ & $10.73 \pm 0.49^{a}$ & $89.78 \pm 0.54$ & $f$ & $5.61 \pm 0.24 \mathrm{a}$ & $20.97 \pm 0.54$ & c & $5.61 \pm 0.24 \mathrm{a}^{\mathrm{a}}$ & $19.89 \pm 0.54$ & c & $5.61 \pm 0.73$ a & $17.74 \pm 0.54$ & b \\
\hline $4 d$ & $10.24 \pm 0.49^{a}$ & $34.95 \pm 0.54$ & d & $6.10 \pm 0.24$ & $16.67 \pm 0.54$ & t & $5.85 \pm 0.49 \mathrm{a}$ & $19.89 \pm 0.54$ & c & $5.85 \pm 0.49$ & $17.74 \pm 0.54$ & b \\
\hline $7 \mathrm{~d}$ & $10.49 \pm 0.24^{a}$ & $25.27 \pm 0.54$ & & $5.61 \pm 0.24 \mathrm{a}^{\mathrm{a}}$ & $18.82 \pm 0.54$ & b & $5.37 \pm 0.49^{a}$ & $19.89 \pm 0.54$ & & $5.61 \pm 0.24^{a}$ & $17.74 \pm 0.54$ & b \\
\hline $9 \mathrm{~d}$ & $10.49 \pm 0.24 \mathrm{a}$ & $25.27 \pm 0.54$ & b & $5.37 \pm 0.49$ & $18.82 \pm 0.54$ & b & $5.61 \pm 0.73 \mathrm{a}$ & $15.59 \pm 0.54$ & b & $5.37 \pm 0.49$ a & $15.59 \pm 0.54$ & a \\
\hline $11 \mathrm{~d}$ & $10.49 \pm 0.24$ a & $25.27 \pm 0.54$ & & $5.85 \pm 0.49^{a}$ & $15.59 \pm 0.54$ & a & $5.12 \pm 0.24^{a}$ & $15.59 \pm 0.54$ & b & $5.85 \pm 0.49^{a}$ & $15.59 \pm 0.54$ & a \\
\hline $14 \mathrm{~d}$ & $10.49 \pm 0.00^{a}$ & $25.27 \pm 0.54$ & b & $5.37 \pm 0.49$ & $15.59 \pm 0.54$ & a & $5.37 \pm 0.49 \mathrm{a}$ & $15.59 \pm 0.54$ & b & $5.37 \pm 0.49$ & $15.59 \pm 0.54$ & a \\
\hline $18 \mathrm{~d}$ & $10.59 \pm 0.15$ a & $25.27 \pm 0.54$ & b & $5.61 \pm 0.24 \mathrm{a}$ & $15.59 \pm 0.54$ & a & $5.61 \pm 0.24^{\mathrm{a}}$ & $15.59 \pm 0.54$ & b & $5.37 \pm 0.49 \mathrm{a}$ & $15.59 \pm 0.54$ & a \\
\hline LSD & 0.66 & 2.28 & & 0.74 & 1.13 & & 0.78 & 1.17 & & 0.82 & 0.97 & \\
\hline
\end{tabular}




\subsection{Ag and ZnO NPs excreation}

The excretion kinetics of $\mathrm{Ag}$ and $\mathrm{ZnO}$ nanoparticles were evaluated after 1day of intraperitoneal injection by measuring daily silver and zinc concentrations in feces and urine which collected over 18 days . A considerable mean values of silver and zinc levels in feces and urine were noted after one day of injection (Figure 5, table 5) . with $131.67 \pm 1.36 \mu \mathrm{g} / \mathrm{gm}$ in feces obverse to $2.89 \pm 0.03 \mu \mathrm{g} / \mathrm{ml}$ in urine samples for silver, $214.00 \pm 1.63 \mu \mathrm{g} / \mathrm{gm}$ and $15.00 \pm 0.00 \mu \mathrm{g} / \mathrm{ml}$ for Zinc in feces and urine respectively, these averages decreased with highly statistical significant difference $(p \leq 0.05)$ reaching to the lowest concentrations values after 13 days and stand on $18.33 \pm 1.36 \mu \mathrm{g} / \mathrm{gm}$ in feces sample and extremely low concentration in urine samples with $0.54 \pm 0.03 \mu \mathrm{g} / \mathrm{ml}$, this profile of reducing concentrations over days may be refers to silver binding with the intestinal surfaces which led to decreasing the concentrations in the feces. Silver excretion percentage via urine appeared extremely low $0.8 \pm 0.019 \%$ compared with their percentage in feces $33 \pm 0.17 \%$ (Table 5) as the excretion of Ag NPs via renal system could not be the main path of elimination , furthermore small amount of Ag detected in feces pointed that Ag NPs were excreted slowly by the biliary pathway or deposited in different organs over long time [51].These results approached to the demonstrations of [17] in the recording the fecal excretion percentage of $35.87 \pm 9.94 \%$, also [35] mentioned the recovering of 50\% of silver of infused dose in bile of rat after non oral administration. ZnO NPs excretion kinetic take the same manner as in Ag NPs with the high percentage of fecal excretion 83 $\pm 0.180 \%$ against urinary one with $7.5 \pm 0.020 \%$. As mentioned with silver, the highest excretion averages of zinc in feces and urine samples were recorded after one day of injection $(214 \pm 1.63 \mu \mathrm{g} / \mathrm{gm}, 15 \pm 0.00 \mu \mathrm{g} / \mathrm{ml})$ respectively, and decrees with remark significant differences $(\mathrm{p} \leq 0.05)$ to record their lowest levels in feces and urine samples after 16 days of injection with $65.5 \pm 0.41 \mu \mathrm{g} / \mathrm{gm}, 6.5 \pm 0.41 \mu \mathrm{g} / \mathrm{ml}$ respectively (Figure 4). These high scales of $\mathrm{Zn}$ in feces compared with their equivalents in urine referred to vastly elimination of $\mathrm{ZnO}$ NPs were take placed by the bile into feces [16],[52] which play a crucial roles in the elimination of nanoparticles regardless the factors of experimental animal type and their sex, routes of exposure, particulate size and charge [53].
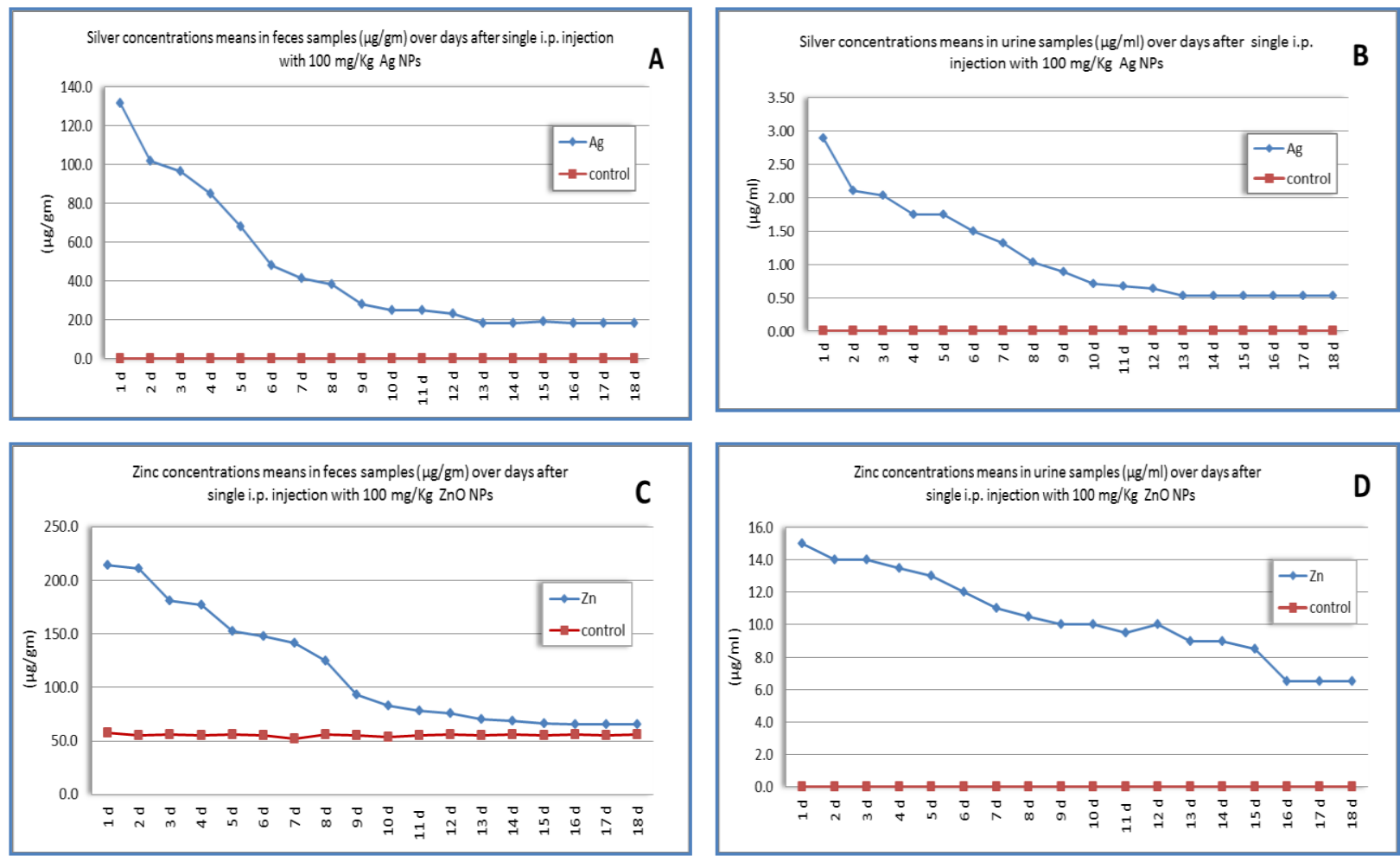

Figure 5. Silver (A,B) and Zinc (C,D) concentrations means \pm SE in feces ( $\mu \mathrm{g} / \mathrm{gm})$ and urine $(\mu \mathrm{g} / \mathrm{ml})$ samples over days after single intraperitoneal (ip) injection with $100 \mathrm{mg} / \mathrm{kg} \mathrm{Ag}$ and $\mathrm{ZnO}$ NPs 
Table 5. silver and zinc concentrations means $\pm \mathrm{SE}$ in feces $(\mathrm{mg} / \mathrm{kg})$ and urine $(\mathrm{mg} / \mathrm{ml})$ over experimental time points after single intraperitoneal injection with $100 \mathrm{mg} / \mathrm{kg} \mathrm{Ag}$ and $\mathrm{ZnO}$ NPs and The excretion amount ( $\mathrm{mg}$ ) and percentage ( $\%$ ) of $\mathrm{Ag}$ and $\mathrm{ZnO}$ NPs via feces and urine

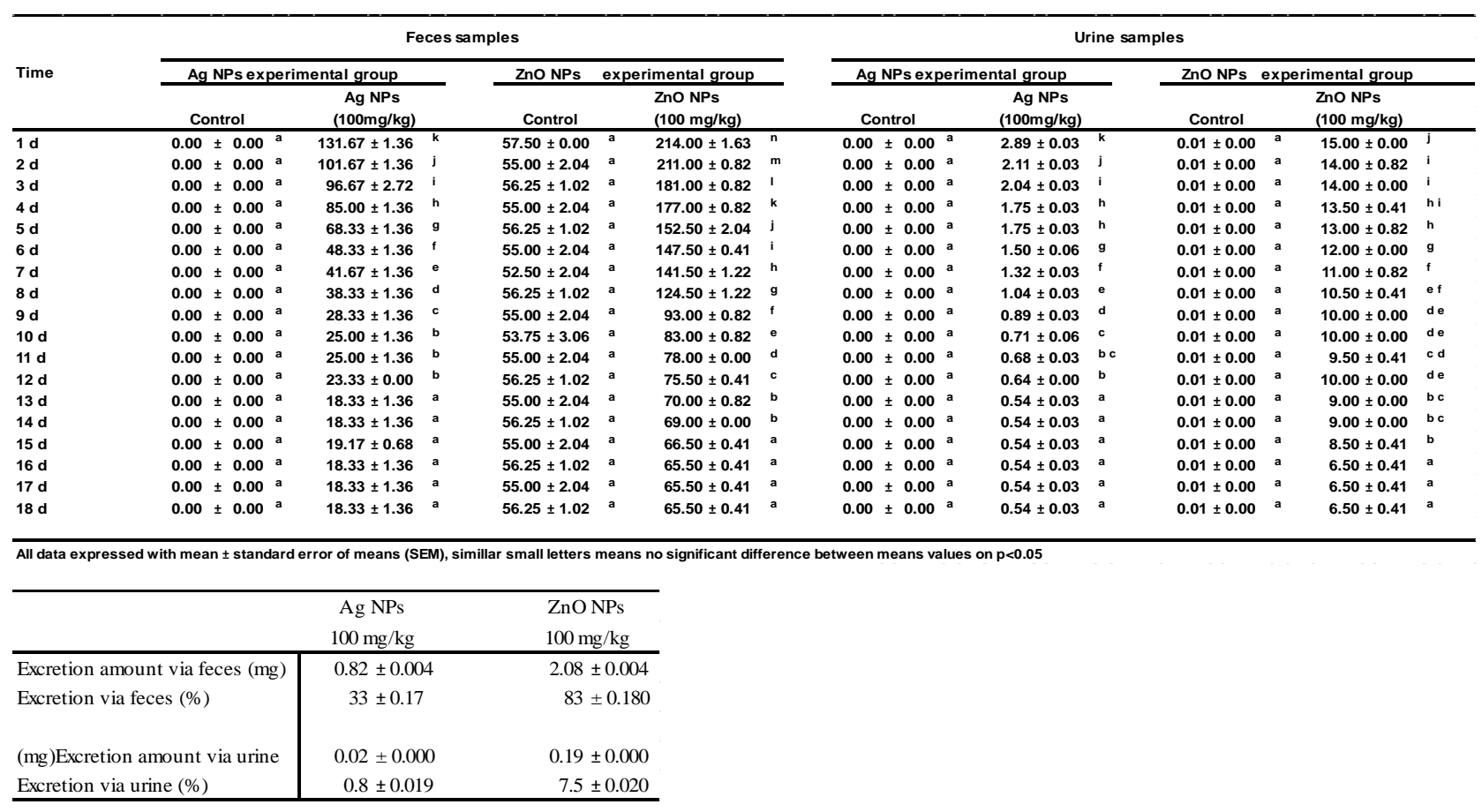

\section{Conclusions}

The present study illustrate the kinetic profile of Ag and ZnO NPs followed the intraperitoneal injection with same dose of the two type of nano particles in mice which is not demonstrated in previous study . In summery ZnO NPs need $30 \mathrm{~min}$ for absorption into the blood and reaching to all studded organs facing to Ag NPs which need $1 \mathrm{~h}$. Ag NPs spend more time for elimination (T1/2 Elem.) with over 8.39 days compared with $\mathrm{ZnO}$ NPs as their lower clearance rate (CL) and higher volume of distribution (Vd) . higher excretion percentage recorded with $\mathrm{ZnO}$ NPs in feces and less by urine compared with Ag NPs .

\section{Acknowledgements}

The authors would like to thank and acknowledge the Materials Research Center- Ministry of and Science and Technology, and the Nutrition Research Institutes -Public Health Directorate - Ministry of health for providing support to carry out this study.

\section{References}

[1] O.Bondarenko, K. Juganson, , A. Ivask, , K. Kasemets, M. Mortimer, and A. Kahru, "Toxicity of Ag, $\mathrm{CuO}$ and $\mathrm{ZnO}$ nanoparticles to selected environmentally relevant test organisms and mammalian cells in vitro: a critical review", Arch. Toxicol., vol.8, pp.1181-1200, 2013.

[2] İ.Y.Enis, Ö.M. Küçükali, H. Sezgin, and E. Ismar, "An Overview of Nanotoxicology”, Engineering Sciences, vol.12, pp.57-65, 2017.

[3] Z.Chen, H.Meng, G. Xing, C.Chen, Y.Zhao, G.Jia, T.Wang, H.Yuan, C.Ye, F.Zhao, Z.Chai, C.Zhu, X.Fang, B. Ma, and L. Wan, "Acute toxicological effects of copper nanoparticles in vivo", Toxicol. Lett, vol.163, pp. 109-120, 2006. 
[4] J. Wang, G. Zhou, C. Chen, H. Yu, T. Wang, Y.Ma, G. Jia, Y. Gao, B. Li, J. Sun, Y. Li, F. Jiao, Y. Zhao, and Z. Chai, "Acute toxicity and biodistribution of different sized titanium dioxide particles in mice after oral administration", Toxicol. Lett. , vol.168, pp.176-185, 2007.

[5] B. Wang, W. Feng, M. Wang, T. Wang, Y. Gu, M. Zhu, H. Ouyang, J. Shi, F. Zhang, Y. Zhao, Z. Chai, H. Wang, and J. Wang, "Acute toxicological impact of nano- and submicro-scaled zinc oxide powder on healthy adult mice", J. Nanopart. Res., vol.10, pp.263-276, 2008.

[6] M. Esmaeillou, M.Moharamnejad, R.Hsankhani, A. A. Tehrani, and H. Maadi, "Toxicity of ZnO nanoparticles in healthy adult mice",Environmental Toxicology and Pharmacology , vol.35, pp.67-71, 2013.

[7] F.Piccinno, F. Gottschalk, S. Seeger, and B.Nowack,'Industrial production quantities and uses of ten engineered nanomaterials for Europe and the world",Journal of Nanoparticle Research, vol.14, pp.1109-1120, 2012.

[8] N.Serpone, D. Dondi, and A. Albini, "Inorganic and organic UV filters: their role and efficacy in sunscreens and suncare products", Inorganica Chimica Acta, vol.360, pp.794-802, 2007.

[9] R. Dastjerdi, and M. Montazer, "A review on the application of inorganic nano-structured materials in the modification of textiles: focus on anti-microbial properties", Colloid Surf Bul, vol.79, pp.5-18, 2010 .

[10] W.Song, J. Zhang, J. Guo, J. Zhang, F. Ding, L. Li, and Z. Sun, "Role of the dissolved zinc ion and reactive oxygen species in cytotoxicity of ZnO nanoparticles" ,Toxicol. Lett, vol.199 ,pp.389-397, 2010.

[11] R.Kumar, M.Chauhan, N. Sharma, and G. R. Chaudhary,"Toxic Effects of Nanomaterials on Environment" . In: Environmental Toxicity of Nanomaterials, edited by: V.Kumar, N.Dasgupta, and S.Ranjan, pp.537,2018.

[12] D.Van der Merwe, R.Gehring, and J. L.Buur, "Toxicokinetics in Veterinary Toxicology", Vetrnary Toxicology, third ed.: basic and clinical principles, pp.133- 243, 2018.

[13] J.E.Riviere,"Pharmacokinetics of nanomaterials: an overview of carbon nanotubes, fullerenes and quantum dots”,Wiley Interdiscip Rev Nanomed Nanobiotechnol , vol.1, pp.26-34, 2009.

[14] M.Baek, H-E. Chung, J. Yu, J-A. Lee, T-H. Kim, J-M. Oh, W-J. Lee, S-M. Paek, J. K Lee, J. Jeong, J-H. Choy, and S-J. Choi "Pharmacokinetics, tissue distribution, and excretion of zinc oxide nanoparticles" International Journal of Nanomedicine , vol.7 , pp.3081-3097, 2012.

[15] Y. Xue, S. Zhang, Y. Huang, T. Zhang, X. Liu, Y. Hu, Z. Zhang, and M.Tang, "Acute toxic effects and gender-related biokinetics of silver nanoparticles following an intravenous injection in mice", J. Appl. Toxicol.,vol. 32, pp.890-899, 2012.

[16] J.Choi, H.Kim, P.Kim, E.Jo, H-M. Kim, M-Y. Lee, S.M. Jin, and K. Park, "Toxicity of Zinc Oxide Nanoparticles in Rats Treated by Two Different Routes: Single Intravenous Injection and Single Oral Administration", Journal of Toxicology and Environmental Health , vol.78, pp.226-243, 2015.

[17] L.Yang, H. Kuang, W. Zhang, Z. P. Aguilar, H. Wei , and H. Xu, "Comparisons of the biodistribution and toxicological examinations after repeated intravenous administration of silver and gold nanoparticles in mice", Scientific Reports, vol.7, pp.1- 12, 2017. 
[18] J.S. Taurozzi, V.A.Hackley, and M.R. Wiesner (Eds),"Preparation of nano particle dispersions from powderd material using ultrasonic dispersion”, National Institute of Standard and Technology (NIST), vol.1.1, pp.1-15, 2012.

[19] EPA "Method 3052. Microwave assisted acid digestion of siliceous and organically based matrices, SW-846", Environmental Protection Agency ,pp.1-20,1996.

[20] S. J. Mangum,"Microwave Digestion . EPA Method 3052 on the Multiwave 3000. PerkinElmer Inc. USA, pp.1-3, 2009.

[21] M. Zhu, S. Perrett, and G. Nie, "Understanding the Particokinetics of Engineered Nanomaterials for Safe and Effective Therapeutic Applications", small , vol.9, pp.1619-1634, 2013.

[22] R. S.Yang, L. W.Chang, J. P.Wu, M. H. Tsai, H. J.Wang, Y.C.Kuo, T. K. Yeh, C. S. Yang, and P. Lin Environ. Health Perspect, vol.115, pp.1339-1343, 2007.

[23] X. Feng, A. Chen, Y. Zhang, J. Wang, L. Shao, and L.Wei,"Central nervous system toxicity of metallic nanoparticles”, Int. J. Nanomed, vol.10, pp.4321-4340, 2015.

[24] M.F.Flessner,"The transport barrier in intraperitoneal therapy”,Am J Physiol Renal Physiol, vol.288,pp.433-442, 2005.

[25] N. Lewinski, V. Colvin, and R. Drezek, “Cytotoxicity of nanoparticles”, Small, vol.4, pp.26-49,2008.

[26] M. Nowacki, M. Peterson, T. Kloskowski, E. McCabe, D. C. Guiral, K. Polom, K. Pietkun, B. Zegarska, M. Pokrywczynska, T. Drewa, F. Roviello, E. A. Medina, S. L. Habib, and W. Zegarski, "Nanoparticle as a novel tool in hyperthermic intraperitoneal and pressurized intraperitoneal aerosol chemotheprapy to treat patients with peritoneal carcinomatosis" ,Oncotarget, vol.8, pp.78208-78224, 2017.

[27] J.K.Leypoldt, "Solute Transport Across the Peritoneal Membrane",Journal of the American Society of Nephrology ,vol.13, pp.84-91, 2002.

[28] K. Park, E.J. Park, I.K. Chun, K. Choi, S.H. Lee, J.Yoon , and B.C.Lee, "Bioavailability and toxicokinetics of citrate-coated silver nanoparticles in rats" , Arch Pharm Res , vol.34, pp.153-158, 2011.

[29] C-H. Li, C-C. Shen, Y-W. Cheng, S-H. Huang, C-C. Wu, C-C. Kao, J-W. Liao, and J-J. Kang, "Organ biodistribution, clearance, and genotoxicity of orally administered zinc oxide nanoparticles in mice", Nanotoxicology ,vol. 6, pp.746-756, 2012.

[30] G.Oberdorster, E. Oberdorster, and J.Oberdorster, "Nanotoxicology: an emerging discipline evolving from studies of ultrafine particles", Environ Health Perspect., vol.113, pp.823-839, 2005.

[31] W. I. Hagens, A.G. Oomen, W. H. De Jong, F. R. Cassee, and A. J.A.M. Sips ,'What do we (need to) know about the kinetic properties of nanoparticles in the body?", Regulatory Toxicology and Pharmacology, vol.49, pp.217-229, 2007.

[32] F. K. Swirski, M. Nahrendorf, M. Etzrodt, M. Wildgruber, V. C. Retamozo, P. Panizzi, J-L. Figueiredo, R. H. Kohler, A. Chudnovskiy, P. Waterman, E. Aikawa, T. R. Mempel, P. Libby, R.Weissleder ,and M. J. Pittet, , "Identification of splenic reservoir monocytes and their deployment to inflammatory sites”, Science, vol.325, pp.612-616,2009. 
[33] M.B.Genter, N.C.Newman, H.G. Shertzer, S.F. Ali, and B. Bolon,"'Distribution and systemic effects of intranasally administered $25 \mathrm{~nm}$ silver nanoparticles in adult mice", Toxicol Pathol, vol. 40 , p. 10041013, 2012.

[34] H. M.Braakhuis, I.Gosens, P.Krystek, J.Boere, F.R. Cassee, P.Fokkens, J.Post, H.Van Loveren, and M.Park, "Particle size dependent deposition and pulmonary inflammation after short-term inhalation of silver nanoparticles", Part. Fibre Toxicol , vol.11, pp. 1-16, 2014.

[35] N.Hadrup, and H. R. Lam,"Oral toxicity of silver ions, silver nanoparticles and colloidal silver - A review", Regulatory Toxicology and Pharmacology , vol.68, pp.1-7, 2014.

[36] R. Wen, L. Hu, G. Qu, Q. Zhou, and G. Jiang, "Exposure, tissue biodistribution, and biotransformation of nanosilver", NanoImpact , vol. 2 , pp.18-28, 2016.

[37] G.Tarantino, A.Scalera, and C.Finelli,'Liver-spleen axis: Intersection between immunity, infections and metabolism", World J Gastroenterol , vol.19, pp.3534-3542, 2013.

[38] K.H.Chen, D. J.Lundy, E.K.-W. Toh, C.-H. Chen, C.Shih, P.Chen, H.-C. Chang, J. J. Lai, P. S. Stayton, A. S.Hoffman, and P. C.-H. Hsieh, "Nanoparticle Distribution during Systemic Inflammation is Size-Dependent and Organ-Specific", Nanoscale. Royal Society of Chemistry ,vol.10, 2015.

[39] A. Nel, T. Xia, L.Madler, and N. Li,'Toxic potential ofmaterials at the nanolevel", Science, vol.311, pp.622-627, 2006.

[40] Y. Gao, X. Zhu, Y. Zhang, X. Chen, L. Wang, W. Feng, C. Huang, and F, Li, "In vivo biodistribution and passive accumulation of upconversion nanoparticles in colorectal cancer models via intraperitoneal injection”, RSC Advances, vol.7 ,pp. 31588-31596 , 2017.

[41] Z. Lu, J. Wang, M. G. Wientjes, and J. L-S. Au, "Intraperitoneal therapy for peritoneal cancer", Future Oncol, vol.6, pp.1625-1641, 2010.

[42] S. H. Cheng, F.C.Li, J. S. Souris, C.-S. Yang, F. G. Tseng, H. S. Lee, C. T. Chen, C. Y. Dong, and L.W.Lo, "Visualizing Dynamics of Sub Hepatic Distribution of Nanoparticles Using Intravital Multiphoton Fluorescence Microscopy",ACS Nano, vol.6, pp.4122-4131, 2012.

[43] A.M. Gatti, "Biocompatibility of micro-and nano-particles in the colon", Biomaterials, vol.25, pp.385392, 2004.

[44] K. Dziendzikowska, J. Gromadzka-Ostrowska, A. Lankoff , M. Oczkowski, A. Krawczyńska, J. Chwastowska, M. Sadowska-Bratek, E. Chajduk, M. Wojewódzka, M. Du_sinsk, and M. Kruszewski "Time-dependent biodistribution and excretion of silver nanoparticles in male Wistar rats" , J Appl Toxicol, vol.32, pp.920-928, 2012.

[45] J. H. Lee, J. H. Sung, H. R. Ryu, K. S. Song, N. W. Song, H. M. Park, B. S. Shin, K. Ahns, M. Gulumian, E. M. Faustman, and I.J.Yu ,'Tissue distribution of gold and silver after subacute intravenous injection of co-administered gold and silver nanoparticles of similar sizes" ,Archives of Toxicology, vol.92, pp.1393-1405, 2018.

[46] H.-J. Paek, Y.-J.Lee, H.-E.Chung, N.-H.Yoo, J.-A.Lee, M.-K.Kim, J.K. Lee, J. Jeong, and S.-J. Choi, "Modulation of the pharmacokinetics of zinc oxide nanoparticles and their fates in vivo",Nano scale , vol.5, pp.11416-11427, 2013.

[47] D.P.K. Lankveld, A.G. Oomen, P. Krystek, A. Neigh, A.T-d Jong, C.W. Noorlander, J.C.H. Van Eijkeren, R.E. Geertsma, and W.H. De Jong "The kinetics of the tissue distribution of silver nanoparticles of different sizes",Biomaterials, vol.31, pp.8350-8361, 2010. 
[48] A.N. Ananth, S. C Daniel, G. K., T. A. Sironmani, and S. Umapathi, "PVA and BSA stabilized silver nanoparticles based surface-enhanced plasmon resonance probes for protein detection" Colloids and Surfaces B. Biointerfaces, vol.85, pp.138-144, 2011.

[49] K.Pfurtscheller, T. Petnehazy, W. Goessler, V. Bubalo, L.P. Kamolz, and M. Trop,” Transdermal uptake and organ distribution of silver fromtwo different wound dressings in rats after a burn trauma" ,Wound Repair Regen, vol.22 ,pp. 654-659, 2014.

[50] M.Van der Zande, R.J.Vandebriel, E.Van Doren, E. Kramer, Z.H. Rivera, C.S. Serrano-Rojero, E.R. Gremmer, J.Mast, R.J. Peters, P.C.Hollman, P.J.Hendriksen, H.J.Marvin, A.A.Peijnenburg, and H. Bouwmeester, "Distribution, elimination, and toxicity of silver nanoparticles and silver ions in rats after 28-day oral exposure", ACS Nano , vol.6, pp.7427-7442, 2012.

[51] Y.Zhang, T. R. Nayak, H. Hong, and W. Cai, "Biomedical Applications of Zinc Oxide Nanomaterials", Curr Mol Med, vol.13 ,pp. 1633-1645, 2013.

[52] W-S. Cho, B-C. Kang, J. K. Lee, J. Jeong, J-H. Che, and S. H. Seok, "Comparative absorption, distribution, andexcretion of titanium dioxide and zinc oxidenanoparticles after repeated oral administration", Particle and Fibre Toxicology, vol. 10, pp.1-9, 2013.

[53] S-J. Choi, and J-H. Choy, "Biokinetics of zinc oxide nanoparticles: toxicokinetics, biological fates, and protein interaction", International Journal of Nanomedicine , vol.9, pp. 261-269, 2014.

[54] I. Kashi1, and K. Moussaceb, "New mathematical models for predicting the lifetime of EPDM insulators: Effect of elongation at break on the kinetic degradation of EPDM insulators subjected to thermo-oxidation”, Periodicals of Engineering and Natural Sciences, vol.6, pp.71-88, 2018.

[55] S. Kumar, D.Jayadevappa, and M.V. Shetty, "A Novel approach for Segmentation and Classification of brain MR Images using Cluster Deformable Based Fusion Approach", Periodicals of Engineering and Natural Sciences, vol.6, pp.237-242,2018.

[56] K. ALTUNTAS, E. DEBIK, D. Kozal , and I. I. YORUK, "Adsorption of Copper Metal Ion from Aqueous Solution by Nanoscale Zero Valent Iron (nZVI) Supported on Activated Carbon", Periodicals of Engineering and Natural Sciences, vol.5, pp.61-64, 2017.

[57] N. A. Mutua, A. B. Ouma, and O. S. Atieno, "Kinetic Study of the Thermal Decomposition for Mixed Municipal Solid Waste Using Thermogravimetric Analysis", Periodicals of Engineering and Natural Sciences, vol.5, pp.355-363,2017.

[58] J. Šutković and A.Jašarević, "A review on Nanoparticle and Protein interaction in biomedical applications", Periodicals of Engineering and Natural Sciences, vol.4, pp.34-40,2016. 